\section{Reprodutibilidade de um questionário de consumo alimentar para crianças em idade escolar}

\section{Reproducibility of a typical day food choices questionnaire for schoolchildren}

Adriana Soares Lobo 1

Maria Alice Altenburg de Assis 2

Mauro Virgílio Gomes de Barros 3

Maria Cristina Marino Calvo 4

Sérgio Fernando Torres Freitas 5

\author{
1 Departamentos de Nutrição. Universidade do Extremo Sul \\ Catarinense. Criciúma, SC, Brasil. \\ 2 Programa de Pós Graduação em Nutrição. Universidade Federal \\ de Santa Catarina. Campus Universitário. Trindade. CEP: 88.040- \\ 970. Florianópolis, SC, Brasil. E-mail: massis@ccs.ufsc.br \\ 3 Grupo de Pesquisa em Estilos de Vida e Saúde. Programa de Pós- \\ Graduação em Hebiatria. Escola Superior de Educação Física. \\ Universidade de Pernambuco. Recife, PE, Brasil. \\ 4,5 Programa de Pós Graduação em Saúde Pública. Universidade \\ Federal de Santa Catarina. Campus Universitário. Florianópolis, \\ SC, Brasil.
}

\section{Resumo}

Objetivos: avaliar a reprodutibilidade de um questionário para obter auto-relato de 16 alimentos consumidos em cinco refeições num dia típico da semana, em escolares do ensino fundamental.

Métodos: participaram do estudo 342 escolares das primeiras às quartas séries do ensino fundamental (duas escolas públicas e três privadas) de Florianópolis, Santa Catarina, Brasil. A reprodutibilidade foi determinada através da comparação das respostas obtidas em dois dias, após um intervalo de três dias. Análises foram estratificadas por série escolar (primeiras e segundas versus terceiras e quartas) e rede escolar (pública $e$ privada), para determinar, o percentual de concordância (\%CC) e estatística kappa ajustada.

Resultados: nas escolas públicas, os valores de \%CC variaram entre $69 \%$ (pães) e $84,5 \%$ (peixes) e os valores de kappa entre 0,38 (pães) e 0,69 (peixes). Nas escolas privadas os \%CC variaram entre $73,9 \%$ (pães) e $91,6 \%$ (feijão) e os valores de kappa entre 0,48 (pães) e 0,83 (feijão). Maiores valores de \%CC e de kappa foram obtidos para as terceiras e quartas séries do que para as primeiras e segundas, nas escolas públicas e privadas.

Conclusões: a reprodutibilidade do questionário foi demonstrada para os escolares de escolas privadas $e$ públicas, embora melhores resultados foram obtidos nas escolas privadas.

Palavras-chave Questionário, Consumo alimentar, Escolares, Reprodutibilidade 


\section{Introdução}

Evidências científicas têm sugerido que o estilo de vida na infância pode exercer um efeito importante sobre o risco de desenvolvimento de doenças crônicas não transmissíveis (DCNT) na vida adulta, tais como obesidade, doença arterial coronariana, hipertensão arterial, diabetes e certos tipos de câncer. ${ }^{1-3} \mathrm{~A}$ prevalência da obesidade entre as crianças de idade escolar está aumentando no Brasil. Os dados de duas pesquisas nacionais realizadas em 1975 e 1997 mostraram que a prevalência de sobrepeso triplicou em crianças brasileiras de seis a nove anos de idade (4,9\% em 1975; 17,4\% em 1997). ${ }^{4}$

Para melhor elucidar a relação entre a alimentação e a obesidade, bem como avaliar a efetividade de programas de intervenção nutricional, é necessário dispor de métodos válidos de coleta de dados de consumo alimentar, que possam ser aplicados em pesquisas populacionais. 5

Umas das principais dificuldades para realização de estudos de abrangência populacional de consumo alimentar de crianças em idade escolar é a escassez de instrumentos adequados e razoavelmente precisos de coleta de dados.6,7 Em crianças, estes problemas têm como agravante a limitação cognitiva delas para relatarem a ingestão de alimentos, incluindo um inadequado desenvolvimento do conceito de tempo e quantidade, memória e atenção insuficientes e o desconhecimento do nome dos alimentos e das preparações. 6

Para a realização de estudos transversais de abrangência populacional a utilização de questionários parece ser uma opção viável, devido ao seu baixo custo e facilidade de administração. ${ }^{7}$

Apesar de existirem instrumentos dietéticos desenvolvidos e validados com crianças de outros países, são raros os estudos de reprodutibilidade e validade de questionários no Brasil com escolares na faixa etária de sete a dez anos de idade. ${ }^{8}$

O objetivo do presente estudo foi verificar a reprodutibilidade do Questionário Dia típico de atividade física e de consumo alimentar (DAFA - seção alimentar) para escolares das primeiras às quartas séries do ensino fundamental de escolas públicas e privadas e comparar os dados entre as séries e as escolas. Estudos de reprodutibilidade servem como premissas da eficiência do instrumento, para depois então o instrumento ser submetido à validação. ${ }^{9}$

\section{Métodos}

O questionário DAFA foi um dos instrumentos de coleta de dados de uma pesquisa de maior abrangência, realizada em Florianópolis, Santa Catarina, Brasil, no ano de 2002, com o objetivo de avaliar a prevalência de sobrepeso e obesidade em escolares de sete a dez anos de idade. 10

$\mathrm{O}$ instrumento foi desenvolvido para as crianças da primeira à quarta série do ensino fundamental. $\mathrm{O}$ questionário é um instrumento ilustrado e estruturado, delineado para obter informações sobre as escolhas de cinco refeições e 16 grupos de alimentos e itens alimentares representativos de um dia típico de semana. Como requisitos importantes na formatação do questionário foram levados em consideração: a fase curricular e cognitiva das crianças na faixa etária considerada; a viabilidade de aplicação ao conjunto de alunos em sala de aula; a padronização de um único instrumento para os alunos das primeiras às quartas séries; a apresentação atraente $\mathrm{e}$ a facilidade de aplicação em sala de aula, por pessoal treinado e com a supervisão do professor; a clareza das informações requeridas e o baixo custo. 8

O delineamento do questionário foi realizado a partir de várias sessões de estudos e discussões, com a participação de profissionais das áreas de nutrição (nutricionistas e pediatras), pedagogia e design gráfico. Para a inclusão dos alimentos e grupos de alimentos foram consideradas as recomendações alimentares para as crianças de sete a dez anos de idade, 11,12 os dados de pesquisas sobre os hábitos alimentares de escolares no Brasil,13-15 e os alimentos oferecidos pela merenda escolar nas escolas públicas de Florianópolis.

A primeira versão do instrumento foi testada em estudo piloto, conduzido junto a um grupo de escolares da primeira à quarta série do ensino fundamental de uma escola da rede pública federal de Florianópolis, para avaliar a sua validade e reprodutibilidade. 8 O instrumento foi refinado a partir desse estudo piloto e a estrutura da versão final ficou distribuída em três páginas, com 72 ilustrações coloridas. A criação dos símbolos e desenhos foi realizada por uma empresa de design gráfico.

Foram convidados a participar do presente estudo todos os escolares das primeiras às quartas séries de duas escolas públicas e três escolas privadas do município de Florianópolis. As escolas foram selecionadas intencionalmente para garantir a presença de escolares de diferentes níveis socioeconômicos. Dos 389 alunos convidados, 378 receberam consentimento dos pais, através da assinatura do termo de consentimento. Destes, 355 participaram do estudo (compareceram nos dias de aula em que foram aplicados o teste e o re-teste do questionário). A amostra final foi de 342 alunos entre seis 
e dez anos de idade. Os dados de oito alunos com mais de onze anos de idade foram excluídos das análises devido ao baixo número de representantes dessa faixa etária.

As características socioeconômicas dos participantes foram obtidas através de um questionário enviado aos pais, sendo incluídas questões estruturadas que permitissem a classificação econômica através do critério de classificação econômica do Brasil da Associação Nacional de Empresas de Pesquisa (ANEP). ${ }^{16}$

Os dados foram coletados entre os meses de março e junho de 2003. O protocolo da pesquisa foi submetido e aprovado pelo Comitê de Ética em Pesquisa com Seres Humanos da Universidade Federal de Santa Catarina e os pais ou responsáveis assinaram o termo de consentimento livre e esclarecido.

Foram obtidos os dados da massa corporal e da estatura, seguindo-se os procedimentos recomendados pela Organização Mundial de Saúde. ${ }^{17}$ As medidas de massa corporal foram realizadas através de balança digital da marca Filizola, com escalas de 100 gramas. Para mensuração da altura, utilizou-se uma fita métrica milimetrada, aderida à parede, com o ponto zero da fita aderida ao nível do solo. Os escolares foram mensurados descalços, usando roupas leves.

O Índice de Massa Corporal (IMC), computado através da massa corporal em quilogramas $(\mathrm{Kg})$ dividido pela estatura em metros quadrados $\left(\mathrm{m}^{2}\right)$, foi utilizado para a classificação das crianças em baixo peso, sobrepeso ou obesidade. Foram utilizados os pontos de corte do IMC publicados por Must et al., 18 recomendados pela World Health Organization (WHO). 17 Cinco idades foram definidas: seis anos (6,0-6,9 anos), sete anos (7,0-7,9 anos), oito anos $(8,0-8,9)$, nove anos $(9,0-9,9)$ e dez anos $(10,0$ a 10,9 anos). Os pontos de corte selecionados foram os correspondentes ao valor da metade do ano, por exemplo, o ponto de corte de 7,5 anos para a idade de sete anos.

O questionário foi aplicado em sala de aula como um exercício supervisionado, seguindo um protocolo padronizado. Esse protocolo foi construído a partir de um estudo piloto conduzido com 21 escolares da primeira série do ensino fundamental de uma escola da rede municipal de ensino público de Florianópolis. O questionário era distribuído a todos os escolares e a eles era solicitado que assinalassem todos os alimentos que consumiam em cada uma das cinco refeições, em um dia típico de semana. O aplicador orientava o preenchimento do instrumento, com linguajar apropriado, fazendo uso de um questionário em formato de pôster. Dois auxiliares e o

\section{Figura 1}

Página da seção de consumo alimentar do Questionário Dia Típico de Atividades Físicas (DAFA) representando refeições com os 16 grupos de alimentos.

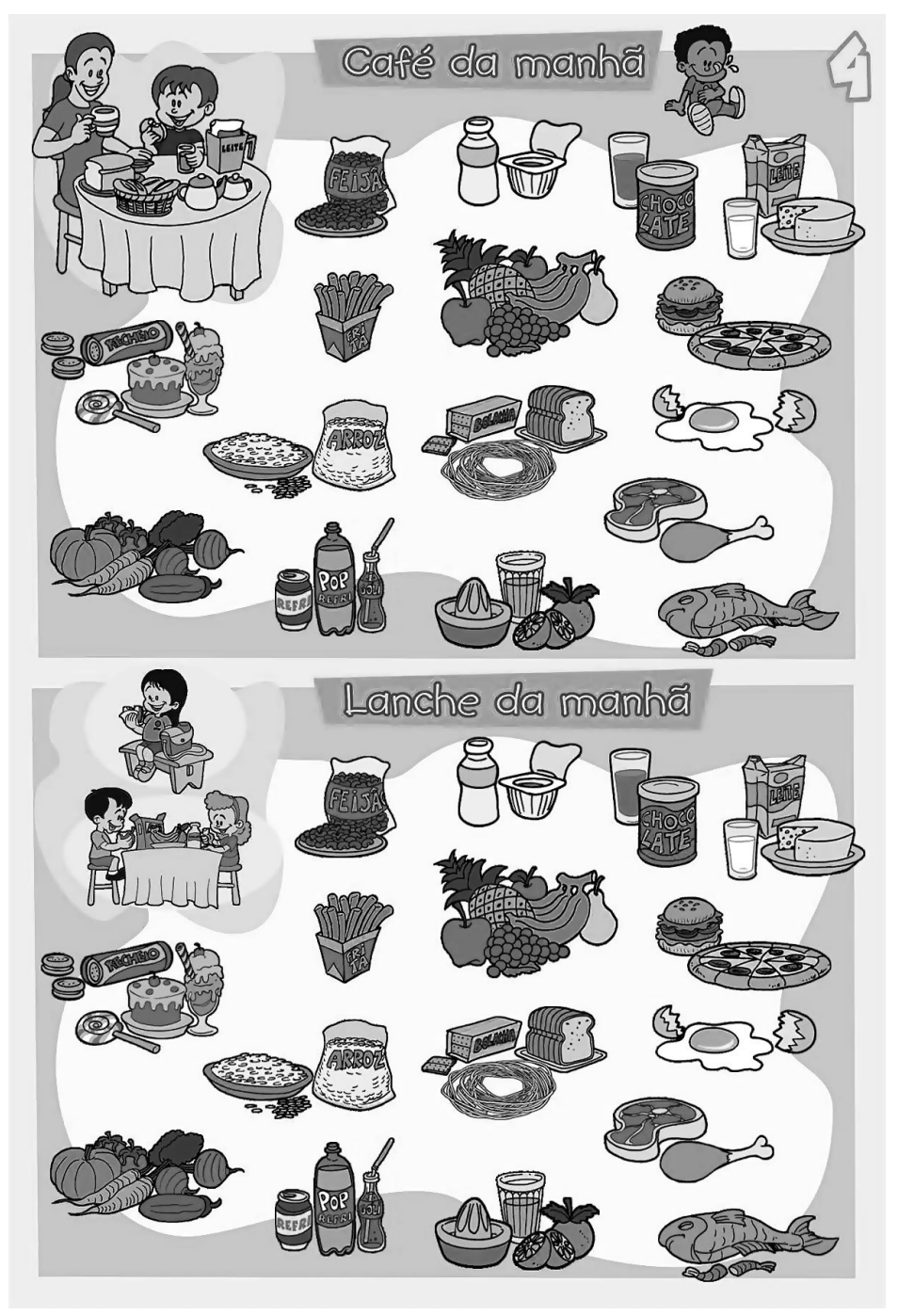

professor circulavam pela classe para responder a eventuais dúvidas e encorajar a resposta das crianças. A aplicação foi realizada antes do lanche escolar e levava em média de 25 a 35 minutos.

A consistência das medidas de alimentação obtidas através do questionário foi testada em duas aplicações (T1 e T2) do instrumento ao mesmo grupo de escolares. O intervalo entre as aplicações, realizadas na terça e na sexta-feira, foi de três dias.

As freqüências de baixo peso, sobrepeso e obesidade foram computadas para os meninos e as meninas, nas escolas públicas e privadas. Comparações das freqüências entre os escolares da rede pública e privada foram calculadas através do teste do quiquadrado. 
Para a análise da reprodutibilidade teste/re-teste do questionário, os dados de consumo obtidos em cada uma das cinco refeições foram agrupados. A comparação entre as duas aplicações foi efetuada item a item, por rede de ensino (público e privado) e séries (primeiras e segundas séries e terceiras e quartas séries), por meio de duas medidas: índices de concordância relativa (\%CC); e estatística kappa ajustada (\% de concordância além daquela esperada pela chance). Devido às assimetrias na distribuição dos dados na tabela de contingência que comprometem a interpretação e cálculo de índices kappa, recorreu-se à determinação de um índice kappa ajustado, conforme sugerido na literatura. ${ }^{19} \mathrm{O}$ índice kappa diferencia-se da concordância relativa em virtude de ser um índice ajustado à chance estatística de concordância devida ao acaso. Em geral os valores de kappa variam de zero $(0=$ ausência de concordância) a um (1=concordância perfeita), mas valores negativos são possíveis. Conforme sugerido na literatura, foram adotados os seguintes critérios na interpretação dos valores de kappa: pobre $(\mathrm{k} \leq 0,20)$; fraco $(0,21 \leq k \leq 0,40)$; moderado $(0,41 \leq k \leq 0,60)$ e substancial $(\mathrm{k}>0,60) .20$

As análises foram realizadas através do programa SPSS 10.0 e de uma planilha eletrônica desenvolvida por Mackinnon, ${ }^{21}$ para a obtenção do kappa ajustado.

\section{Resultados}

A Tabela 1 descreve as características sóciodemográficas e o estado nutricional das crianças das escolas públicas e privadas. Quase a metade dos escolares da rede pública pertenciam à classe

Tabela 1

Características sócio-demográficas e estado nutricional dos escolares, segundo a rede de ensino.

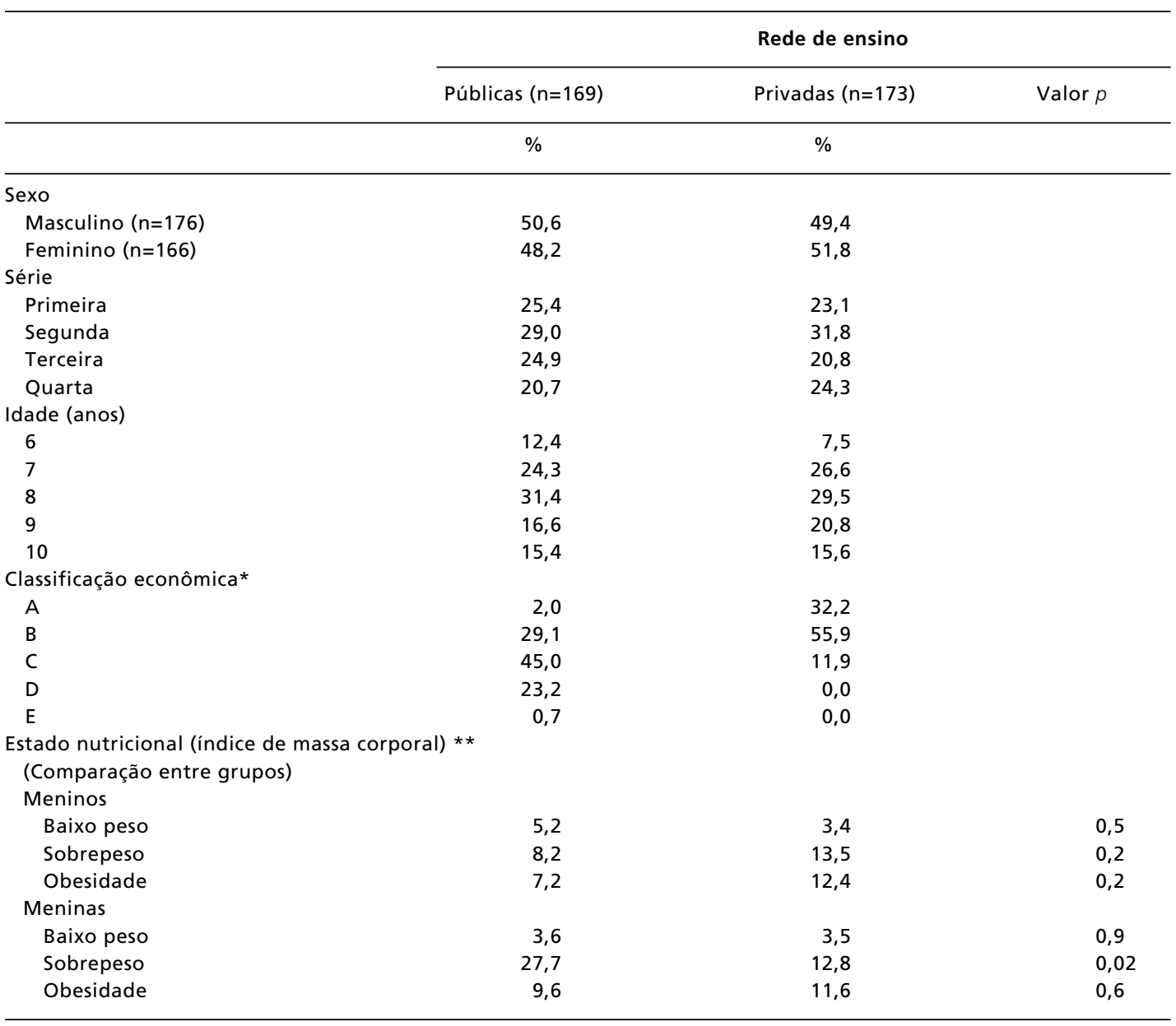

* ANEP (Associação Nacional de Empresas de Pesquisa). Critério de classificação econômica Brasil;16 ; ** Pontos de corte de índice de massa corporal, segundo Must et al. Reference data for obesity $85^{\text {th }}$ and $95^{\text {th }} \ldots$ Am J Clin Nutr. 1991; 53: 839-46.18 
Tabela 2

Reprodutibilidade teste (T1) e re-teste (T2) do questionário de consumo alimentar nas escolas públicas, segundo a série.

\begin{tabular}{|c|c|c|c|c|c|c|c|c|c|c|c|c|}
\hline \multirow[t]{4}{*}{ Alimentos } & \multicolumn{12}{|c|}{ Série escolar } \\
\hline & \multicolumn{4}{|c|}{ Todas as séries } & \multicolumn{4}{|c|}{ Primeira e Segunda } & \multicolumn{4}{|c|}{ Terceira e Quarta } \\
\hline & \multicolumn{2}{|c|}{$\%$ consumo } & \multirow[t]{2}{*}{$\% \mathrm{CC}^{*}$} & \multirow[t]{2}{*}{ kappa } & \multicolumn{2}{|c|}{$\%$ consumo } & \multirow[t]{2}{*}{$\% C C^{*}$} & \multirow[t]{2}{*}{ kappa } & \multicolumn{2}{|c|}{$\%$ consumo } & \multirow[t]{2}{*}{$\% \mathrm{CC}^{*}$} & \multirow[t]{2}{*}{ kappa } \\
\hline & $\mathrm{T} 1$ & $\mathrm{~T} 2$ & & & T1 & $\mathrm{T} 2$ & & & $\mathrm{~T} 1$ & $\mathrm{~T} 2$ & & \\
\hline Feijão & 40,2 & 42,7 & 82,1 & 0,64 & 37,4 & 37,8 & 77,8 & 0,56 & 43,6 & 48,6 & 87,3 & 0,75 \\
\hline Achocolatado & 18,5 & 17,0 & 82,5 & 0,65 & 20,0 & 15,2 & 80,9 & 0,62 & 16,6 & 19,2 & 84,4 & 0,69 \\
\hline logurte & 38,1 & 35,9 & 77,6 & 0,55 & 36,1 & 32,6 & 73,0 & 0,46 & 40,5 & 39,7 & 83,1 & 0,66 \\
\hline Leite & 20,0 & 19,5 & 83,9 & 0,68 & 18,3 & 17,2 & 82,4 & 0,65 & 22,1 & 22,3 & 85,7 & 0,71 \\
\hline Doces & 27,8 & 26,4 & 76,1 & 0,52 & 29,8 & 27,0 & 71,1 & 0,42 & 25,5 & 25,7 & 82,1 & 0,64 \\
\hline Batata frita & 18,1 & 14,8 & 82,2 & 0,64 & 19,8 & 13,9 & 81,5 & 0,63 & 16,1 & 15,8 & 83,1 & 0,66 \\
\hline Fruta & 34,3 & 37,9 & 73,0 & 0,46 & 30,4 & 30,2 & 74,6 & 0,49 & 39,0 & 47,0 & 71,2 & 0,42 \\
\hline Pizza, hambúrguer & 20,7 & 14,6 & 83,4 & 0,67 & 22,8 & 12,6 & 81,5 & 0,63 & 18,2 & 16,9 & 85,7 & 0,71 \\
\hline Arroz & 42,2 & 43,6 & 82,6 & 0,65 & 38,7 & 38,0 & 78,5 & 0,57 & 46,5 & 50,1 & 87,5 & 0,75 \\
\hline Pão, macarrão & 54,1 & 55,5 & 69,0 & 0,38 & 43,3 & 46,1 & 66,3 & 0,33 & 67,0 & 66,8 & 72,2 & 0,44 \\
\hline Carne & 24,1 & 22,7 & 80,1 & 0,60 & 22,0 & 17,6 & 81,7 & 0,63 & 26,8 & 28,8 & 78,2 & 0,56 \\
\hline Ovo & 33,4 & 34,8 & 80,1 & 0,60 & 29,8 & 27,2 & 77,0 & 0,54 & 37,7 & 43,9 & 83,9 & 0,68 \\
\hline Verduras & 20,7 & 20,9 & 81,8 & 0,64 & 16,3 & 13,7 & 83,5 & 0,67 & 26,0 & 29,6 & 79,7 & 0,59 \\
\hline Refrigerantes & 33,3 & 29,1 & 73,8 & 0,48 & 35,9 & 30,0 & 68,9 & 0,38 & 30,1 & 28,1 & 79,7 & 0,59 \\
\hline Sucos de frutas & 36,4 & 38,0 & 71,2 & 0,42 & 26,7 & 28,9 & 71,3 & 0,43 & 48,1 & 48,8 & 71,2 & 0,42 \\
\hline Peixe & 21,5 & 19,5 & 84,5 & 0,69 & 20,2 & 14,8 & 83,7 & 0,67 & 23,1 & 25,2 & 85,5 & 0,71 \\
\hline
\end{tabular}

* Concordância relativa.

Tabela 3

Reprodutibilidade teste ( $\mathrm{T} 1)$ e do re-teste $(\mathrm{T} 2)$ do questionário de consumo alimentar nas escolas privadas, segundo a série.

\begin{tabular}{|c|c|c|c|c|c|c|c|c|c|c|c|c|}
\hline \multirow[t]{4}{*}{ Alimentos } & \multicolumn{12}{|c|}{ Série escolar } \\
\hline & \multicolumn{4}{|c|}{ Todas as séries } & \multicolumn{4}{|c|}{ Primeira e Segunda } & \multicolumn{4}{|c|}{ Terceira e Quarta } \\
\hline & \multicolumn{2}{|c|}{$\%$ consumo } & \multirow[t]{2}{*}{$\% \mathrm{CC}^{*}$} & \multirow[t]{2}{*}{ kappa } & \multicolumn{2}{|c|}{$\%$ consumo } & \multirow[t]{2}{*}{$\% C C^{*}$} & \multirow[t]{2}{*}{ kappa } & \multicolumn{2}{|c|}{$\%$ consumo } & \multirow[t]{2}{*}{$\% \mathrm{CC}^{*}$} & \multirow[t]{2}{*}{ kappa } \\
\hline & $\mathrm{T} 1$ & $\mathrm{~T} 2$ & & & T1 & $\mathrm{T} 2$ & & & $\mathrm{~T} 1$ & $\mathrm{~T} 2$ & & \\
\hline Feijão & 23,5 & 23,4 & 91,6 & 0,83 & 25,1 & 23,6 & 90,5 & 0,81 & 21,5 & 23,1 & 92,8 & 0,86 \\
\hline Achocolatado & 21,5 & 19,8 & 84,6 & 0,69 & 20,0 & 21,3 & 83,2 & 0,66 & 23,3 & 17,9 & 86,4 & 0,73 \\
\hline logurte & 29,7 & 30,9 & 85,9 & 0,72 & 30,7 & 30,3 & 84,0 & 0,68 & 28,5 & 31,5 & 88,2 & 0,76 \\
\hline Leite & 20,5 & 22,9 & 84,3 & 0,69 & 21,9 & 23,4 & 82,5 & 0,65 & 19,2 & 20,5 & 88,5 & 0,77 \\
\hline Doces & 30,6 & 28,1 & 79,4 & 0,59 & 33,1 & 29,3 & 76,8 & 0,54 & 27,7 & 26,7 & 82,6 & 0,65 \\
\hline Batata frita & 14,9 & 14,2 & 88,2 & 0,76 & 16,4 & 14,1 & 84,6 & 0,69 & 13,1 & 14,4 & 92,6 & 0,85 \\
\hline Fruta & 30,4 & 28,2 & 82,8 & 0,66 & 29,1 & 27,6 & 81,3 & 0,63 & 32,1 & 29,0 & 84,6 & 0,69 \\
\hline Pizza, hambúrguer & 22,2 & 18,4 & 84,4 & 0,69 & 24,2 & 19,6 & 81,1 & 0,62 & 19,7 & 16,9 & 88,5 & 0,77 \\
\hline Arroz & 26,6 & 25,8 & 91,1 & 0,82 & 26,3 & 24,8 & 89,3 & 0,79 & 26,9 & 26,9 & 93,3 & 0,87 \\
\hline Pão, macarrão & 45,5 & 46,7 & 73,9 & 0,48 & 47,6 & 47,4 & 70,7 & 0,41 & 43,1 & 45,9 & 77,7 & 0,55 \\
\hline Carne & 15,0 & 12,9 & 88,7 & 0,77 & 17,9 & 13,7 & 86,1 & 0,72 & 11,5 & 12,1 & 91,8 & 0,84 \\
\hline Ovo & 27,5 & 25,5 & 86,0 & 0,72 & 27,8 & 24,0 & 82,7 & 0,65 & 27,2 & 27,4 & 90,0 & 0,80 \\
\hline Verduras & 15,5 & 14,1 & 89,4 & 0,79 & 16,2 & 12,6 & 87,6 & 0,75 & 14,6 & 15,9 & 91,5 & 0,83 \\
\hline Refrigerantes & 34,2 & 32,8 & 80,6 & 0,61 & 33,5 & 30,7 & 78,7 & 0,57 & 35,1 & 35,4 & 82,8 & 0,66 \\
\hline Sucos de frutas & 36,9 & 38,6 & 79,1 & 0,58 & 38,3 & 35,8 & 76,8 & 0,54 & 35,1 & 42,1 & 81,8 & 0,64 \\
\hline Peixe & 13,2 & 12,9 & 91,0 & 0,82 & 16,4 & 13,7 & 89,3 & 0,79 & 9,2 & 12,1 & 93,1 & 0,86 \\
\hline
\end{tabular}

* Concordância relativa. 
Resumo da estatística kappa para os testes de reprodutibilidade teste re-teste do questionário de consumo alimentar nas escolas públicas e privadas, segundo a série escolar.

\begin{tabular}{|c|c|c|c|c|c|c|c|}
\hline \multirow[t]{2}{*}{ Valor de kappa } & \multirow{2}{*}{$\begin{array}{l}\text { Interpretação da } \\
\text { concordância* }\end{array}$} & \multicolumn{3}{|c|}{ Escolas públicas } & \multicolumn{3}{|c|}{ Escolas privadas } \\
\hline & & Todas & $\begin{array}{l}\text { Terceiras } \\
\text { séries }\end{array}$ & $\begin{array}{l}\text { Quarta } \\
\text { séries }\end{array}$ & Todas & $\begin{array}{l}\text { Terceiras } \\
\text { séries }\end{array}$ & $\begin{array}{l}\text { Quarta } \\
\text { séries }\end{array}$ \\
\hline$>0,60$ & Substancial & 8 & 7 & 10 & 13 & 12 & 15 \\
\hline $0,41-0,60$ & Moderado & 7 & 7 & 6 & 3 & 4 & 1 \\
\hline $0,20-0,40$ & Fraco & 1 & 2 & 0 & 0 & 0 & 0 \\
\hline
\end{tabular}

* Definido segundo: Petrie A, Sabin C. Medical statitistics at a Glance. London; 2000.20

econômica $\mathrm{C}$, enquanto na rede privada predominaram os escolares das classes A e B. Relativo ao estado nutricional, as freqüências de baixo peso, sobrepeso e obesidade não foram estatisticamente diferentes entre os meninos das escolas públicas e privadas. Entre as meninas, observou-se maior freqüência de sobrepeso nas participantes das escolas públicas quando comparadas às das escolas privadas $(p=0,02)$.

As Tabelas 2 e 3 apresentam os resultados dos testes de reprodutibilidade por item de alimento do questionário, nas escolas públicas e privadas, respectivamente. Os dados foram analisados para todas as séries, e agrupados para as primeiras e segundas séries e terceiras e quartas séries. Foram apresentados o percentual de crianças que relatou ter consumido cada item de alimentos na primeira (T1) e na segunda (T2) aplicação do instrumento, o percentual de concordância $(\% \mathrm{CC})$ e a estatística kappa.

Nas escolas públicas, considerando-se os dados obtidos para o conjunto dos alunos das primeiras às quartas séries, cerca de um terço ou mais dos escolares assinalaram o consumo de oito itens alimentares no teste e no re-teste (feijão, iogurte, frutas, arroz, grupo de pães, ovo, refrigerantes e suco de frutas). Os outros oito itens alimentares foram relatados por cerca de $15 \%$ a $28 \%$ dos escolares. Os valores de percentual de concordância variaram entre $69 \%$ (grupo de pães) e $84,5 \%$ (peixes) e os valores de kappa entre 0,38 (grupo de pães) e 0,69 (peixes). Quando as análises foram estratificadas segundo as séries, observaram-se maiores valores de percentual de concordância e da estatística kappa para as terceiras e quartas séries do que para as primeiras e segundas séries.

Nas escolas privadas, as análises para todas as séries mostraram que três itens alimentares foram assinalados por mais de um terço dos escolares (grupo de pães, refrigerantes e suco de frutas), nove itens por cerca de $18 \%$ a $30 \%$ (feijão, achocolatado, iogurte, leite, doces, frutas, pizza ou hambúrguer, arroz e ovo) e os demais quatro itens foram relatados por cerca de $13 \%$ a $15 \%$ das crianças (batata frita, carne, verduras e peixe). Os percentuais de concordância variaram entre $73,9 \%$ (grupo de pães) e $91,6 \%$ (feijão) e os valores da estatística kappa entre 0,48 (grupo de pães) e 0,83 (feijão). Análises estratificadas segundo as séries mostraram a mesma tendência observada nas escolas públicas, isto é, os valores de percentual de concordância e de kappa foram maiores para as terceiras e quartas séries do que para as primeiras e segundas séries.

A Tabela 4 apresenta um resumo dos resultados do estudo de reprodutibilidade do questionário, nas escolas públicas e privadas, segundo a série escolar, mostrando o número de itens alimentares segundo o valor de kappa e a interpretação do índice kappa. Os testes de reprodutibilidade nas escolas privadas apresentaram maiores valores do índice kappa, sendo que 13 dos 16 itens alimentares representados no questionário foram classificados como substanciais. Nas escolas públicas, os testes de reprodutibilidades apontaram oito itens alimentares classificados como substanciais.

\section{Discussão}

Neste estudo conduziu-se uma pesquisa para testar a reprodutibilidade teste/re-teste de um questionário de consumo alimentar construído para crianças das primeiras às quartas séries do ensino fundamental. Entre as limitações do presente estudo, ressalta-se o uso de uma amostra de conveniência, composta por alunos de escolas públicas e privadas do município 
de Florianópolis. Portanto, a generalização dessa pesquisa pode estar limitada, sendo conveniente realizar outros testes de reprodutibilidade quando o questionário for aplicado em outros contextos. No questionário de consumo alimentar, os alimentos foram ilustrados de forma individual ou em grupos, em cinco refeições diárias, e a interpretação dos dados obtidos deve considerar as refeições representadas no questionário e levar em conta que não estão contemplados os alimentos consumidos fora dessas refeições, especialmente após o jantar. Apesar do estudo não ter sido delineado para comparar a reprodutibilidade do questionário de consumo alimentar entre as crianças com sobrepeso e as crianças com peso normal, a amostra analisada apresenta um percentual considerável de crianças em sobrepeso e obesidade $(26,9 \%)$, sendo conveniente que os futuros estudos de validação do questionário considerem a viabilidade de verificar a consistência das respostas segundo o estado nutricional das crianças.

O questionário de consumo alimentar para escolares, apresentado nesse estudo, parece ser o primeiro a ser desenvolvido no Brasil, uma vez que até o presente não foi encontrada na revisão da literatura estudos de reprodutibilidade e validade de questionários dessa natureza para crianças brasileiras de idade escolar. Não foram identificados na literatura estudos de reprodutibilidade e validade de instrumentos de coleta de dados de consumo alimentar que comparassem dados obtidos em escolas públicas e privadas.

O questionário foi bem recebido pelas crianças participantes da pesquisa, as quais demonstraram interesse em respondê-lo. A forma estruturada do questionário contendo ilustrações de alimentos em cinco refeições do dia facilitava a compreensão e as respostas das crianças. Embora os melhores resultados de reprodutibilidade do questionário tenham sido obtidos entre as crianças das escolas privadas, o instrumento mostrou respostas consistentes entre as duas aplicações, em ambas as escolas. Os autores deste trabalho acreditam que o protocolo de aplicação do questionário possa ser mais bem adaptado para a realidade das crianças das escolas públicas, objetivando melhorar a reprodutibilidade do instrumento nessas escolas. Além disto, o questionário pode ser aplicado em sala de aula como um exercício supervisionado, parecendo ser uma opção viável em estudos populacionais.

Apesar da dificuldade em comparar resultados de estudos de reprodutibilidade e validade de questionários de consumo alimentar, em função dos diferentes tipos de variáveis analisadas e dos diferentes métodos de avaliação empregados, as medi- das de concordância da maioria dos itens alimentares mostraram-se similares aos de outros questionários para crianças em idade escolar. $22-26$

Smith et al. ${ }^{24}$ avaliaram, em uma amostra de 365 escolares de sétima série, a reprodutibilidade de um instrumento denominado CATCH Food Checklist (CFC), delineado para obter dados da ingestão de gorduras e sódio de crianças. O CFC consiste de 40 itens de alimentos e grupos de alimentos, onde os avaliados indicam se os consumiram ou não no dia anterior, servindo como uma alternativa de recordatório de 24 horas. Valores de kappa encontrados pelos autores variaram de 0,66 a 0,93 . No entanto, cabe ressaltar que o CFC é um instrumento que tem por objetivo obter dados sobre o consumo alimentar no dia anterior a sua aplicação, minimizando o problema de abstração e memória. Por sua vez, o objetivo do presente estudo foi o de testar a reprodutibilidade do questionário através da obtenção de informações do consumo habitual, o que parece ser uma tarefa cognitiva mais complexa.

Hoelscher et al.25 estudaram a reprodutibilidade e a validade de um questionário, delineado para estudantes americanos do segundo grau, o qual continha perguntas sobre comportamentos "usuais" (de consumo alimentar e de atividade física) e de consumo recente ou relacionado ao dia anterior. Os resultados das análises de reprodutibilidade indicaram a obtenção de respostas mais consistentes, entre o teste e o re-teste, quando as questões especificavam um comportamento recente ou relativo ao dia anterior. Os valores de kappa encontrados variaram de 0,54 a 0,93 para as questões sobre o dia anterior e de 0,41 a 0,79 para as questões sobre os alimentos habitualmente consumidos. Hoelscher et al.25 também observaram que os alimentos claramente definidos no questionário-teste obtiveram melhor validação do que os ingredientes alimentares que compunham as preparações mistas. Portanto, a definição precisa do período e dos alimentos a serem avaliados parece influir nos processos de reprodutibilidade e validade desses instrumentos.

No presente estudo, as respostas das crianças das terceiras e quartas séries forneceram melhores medidas de reprodutibilidade do que as das séries iniciais (primeiras e segundas séries). A maioria dos estudos encontrados na literatura pesquisada foi conduzida com crianças mais velhas do que as participantes no nosso estudo, e freqüentando séries mais avançadas do que as primeiras e segundas séries. No estudo conduzido por Field et al.,26 as medidas de reprodutibilidade (medida pela correlação de Spearman) de ingestão calórica, nutrientes e de alguns alimentos, obtidas por meio de um ques- 
tionário de freqüência de alimentos, se mostraram moderadas (coeficientes de correlação variaram de $0,0$ a 0,76$)$ e foram menores entre escolares de quarta e quinta séries do que entre os de sexta e sétima séries. De acordo com os autores, as crianças mais jovens do estudo apresentavam dificuldade em completar o questionário, o que poderia estar relacionado à dificuldade de abstrair conceitos de médias de ingestão em função do estágio de desenvolvimento cognitivo em que as mesmas se encontravam.

De fato, a idade parece ser um fator que afeta a qualidade do relato do consumo alimentar. Entre as habilidades cognitivas requeridas para o auto-relato do consumo alimentar citam-se um adequado desenvolvimento do conceito de tempo, quantidade em medidas caseiras, boa memória, atenção e conhecimento do nome dos alimentos e preparações. 6

A dificuldade de informar o nome dos alimentos ou de enquadrá-lo em um grupo de alimentos pode ser a razão pela qual, neste e em outros estudos, 25,27 as medidas de concordância tenham variado em função do tipo de alimento presente nos instrumentos avaliados. No presente estudo, itens representados no questionário por apenas um alimento, como o feijão e o arroz, mostraram melhores medidas de concordância, quando comparados a itens que se referem a mais de um alimento, como o do grupo dos pães (ilustrado com pães, macarrão e bolacha salgada).

Destaca-se a importância desse questionário para a avaliação do consumo de frutas e verduras, ali-

\section{Referências}

1. Berenson GS, Srinivasan SR, Nicklas TA. Atherosclerosis: a nutritional disease of childhood. Am J Card. 1998; 26: 229.

2. McGill HC. Childhood nutrition and adult cardiovascular disease. Nutr Rev. 1997; 55: 2-11.

3. Must A. Morbidity and mortality associated with elevated body weight in children and adolescents. Am J Clin Nutr. 1996; 63: 445-7.

4. Wang Y, Monteiro C, Popkin BM. Trends of obesity and underweight in older children and adolescents in the United States, Brazil, China and Russia. Am J Clin Nutr. 2002; 75 : 971-7.

5. McPherson RS, Hoelscher DM, Alexander M, Scanlon KS, Serdula MK. Dietary assessment methods among schoolaged children: validity and reliability. Prev Med. 2000; 31: S11-S33.

6. Livingstone MB, Robson PJ. Measurement of dietary intake in children. Proc Nutr Soc. 2000; 59: 279-93 mentos chaves nos guias e nos programas atuais de intervenção nutricional em escolas. Salienta-se também a possibilidade da obtenção de dados para o arroz e feijão nas refeições escolares, cujo consumo vem decrescendo no Brasil. 15

Apesar da possível inabilidade cognitiva das crianças para responderem sobre consumo habitual, que pode ter levado a desempenhos menos satisfatórios entre as mais jovens, conclui-se no estudo de reprodutibilidade do questionário que o instrumento fornece medidas consistentes sobre o consumo de alimentos. Em suma, em nível de grupo, as crianças das primeiras às quartas séries do ensino fundamental nas escolas da rede pública e privada foram capazes de assinalar os alimentos consumidos num dia típico, através desse questionário de consumo alimentar.

O processo de desenvolvimento de questionários para obter medidas de consumo alimentar pode requerer vários estudos de reprodutibilidade e validade. Diferentes contextos podem requerer o treinamento de aplicadores para a adaptação do protocolo de aplicação. Além disso, estudos de reprodutibilidade servem como premissa para avaliar se o instrumento é de fácil compreensão e se há necessidade de aprimorar o formato do questionário.

Uma vez que foram obtidas boas medidas de reprodutibilidade, nós estamos conduzindo outros estudos, para avaliar a reprodutibilidade e a validade desse questionário sobre medidas do consumo alimentar relativas ao dia anterior, similar ao inquérito recordatório de 24 horas.
7. Baranowski T, Simons-Morton BC. Dietary and physical activity assessment in school-aged children: measurement issues. J School Health. 1991; 61: 195-7.

8. Barros MVG, de Assis MAA, Pires MC, Grosseman S, Vasconcelos FAG, Luna MEP, Honda SS. Validity of physical activity and food consumption questionnaire for children aged seven to ten years old. Rev Bras Saúde Matern Infant. 2007; $7:$ 437-48.

9. Buzzard IM, Stanton CA, Figueiredo M, Fries EA, Nicholson R, Hogan CJ, Danish SJ. Development and reproducibility of a brief food frequency questionnaire for assessing the fat, fiber, and fruit and vegetable intakes of rural adolescents. J Am Diet Assoc. 2001; 101: 1438-46.

10. Assis MA, Rolland-Cachera MF, Grosseman S, Vasconcelos FA, Luna ME, Calvo MC, Barros MV, Pires MM, Bellisle F. Obesity, overweight and thinness in schoolchildren of the city of Florianopolis, Southern Brazil. Eur J Clin Nutr. 2005; 59: 1015-21. 
11. CDC (Centers for Disease Control and Prevention). Guidelines for school health programs to promote lifelong healthy eating. J School Health. 1997; 67: 9-26.

12. Krauss RM, Eckel RH, Howard B, Appel LJ, Daniels SR, Deckelbaum RJ, Erdman JW Jr, Kris-Etherton P, Goldberg IJ, Kotchen TA, Lichtenstein AH, Mitch WE, Mullis R, Robinson K, Wylie-Rosett J, St Jeor S, Suttie J, Tribble DL, Bazzarre TL. AHA dietary guidelines: revision 2000-a statement for healthcare professionals from the Nutrition Committee of the American Heart Association. Circulation. 2000; 102: 2284-99.

13. Caroba DCR. Escolares e professores da rede pública de ensino: análises sobre o consumo alimentar e a influência da propaganda [dissertação mestrado]. Piracicaba: Departamento de Agroindústria, Alimentos e Nutrição, Fundação de Amparo à Pesquisa do Estado de São Paulo, Escola Superior de Agricultura Luiz de Queiroz; 1999.

14. Lobo AS, Lopes AS. Adiposidade corpórea e sua relação com o gasto energético e a composição da dieta de escolares do sexo feminino. Rev Bras Ativ Física Saúde. 2001; 6: 69-78.

15. Monteiro CA. La transición epidemiológica en el Brasil. In: Organización Panamericana de la Salud, Organización Mundial de la Salud, editores. La obesidad en la pobreza: un nuevo reto para la salud pública. Washington, DC; 2000.

16. ANEP (Associação Nacional de Empresas de Pesquisa). Critério de classificação econômica Brasil. Disponível em: http://www.anep.org.br/codigosguias/CCEB.pdf. [2002 nov 20].

17. WHO (World Health Organization). Physical status: the use and interpretation of anthropometry. Geneve; 1995. (Technical Report Series, 854).

18. Must A, Dallal GE, Dietz WH. Reference data for obesity $85^{\text {th }}$ and $95^{\text {th }}$ percentiles of body mass index $\left(\mathrm{w} / \mathrm{ht}^{2}\right)$ and triceps skinfold thickness. Am J Clin Nutr. 1991; 53: 83946.

19. Byrt T, Bishop J, Carlin JB. Bias, prevalence and kappa. J Clin Epidemiol. 1993; 5: 423-9.

Recebido em 3 de agosto de 2006

Versão final apresentada em 31 de outubro de 2007

Aprovado em 7 de novembro de 2007
20. Petrie A, Sabin C. Medical statistics at a Glance. London: Blackwell Science; 2000

21. Mackinnon A. A spreadsheet for the calculation of comprehensive statistics for the assessment of diagnostic tests and inter-rater agreement. Comp Biol Med. 2000; 30: 12734.

22. Andersen LF, Bere E, Kolbjornsen N, Klepp K-I. Validity and reproducibility of self-reported intake of fruit and vegetable among $6^{\text {th }}$ graders. Eur J Clin Nutr. 2004; 58: 771-7.

23. Moore L, Tapper K, Dennehy A, Cooper A. Development and testing of a computerized $24-\mathrm{h}$ recall questionnaire measuring fruit and snack consumption among 9-11 year olds. Eur J Clin Nutr. 2005; 59: 809-816.

24. Smith KW, Hoelscher DM, Lytle LA, Dwyer JT, Nicklas TA, Zive MM, Clesi AL, Garceau AO, Stone EJ. Reliability and validity of the child and adolescent trial for cardiovascular health (CATCH) food checklist: a self-report instrument to measure fat and sodium intake by middle school students. J Am Diet Assoc. 2001; 101: 635-47.

25. Hoelscher DM, Day RS, Kelder SH, Ward JL. Reproducibility and validity of the secondary level schoolbased nutrition monitoring student questionnaire. J Am Diet Assoc. 2003; 103: 186-94.

26. Field AE, Byers T, Hunter DJ, Laird NM, Manson JE, Williamson DF, Willett WC, Colditz GA. Reproducibility and validity of a food frequency questionnaire among fourth to seventh grade innercity school children: implications of age and day-to-day variation in dietary intake. Public Health Nutr. 1999; 2: 293-300.

27. Koehler KM, Cunningham-Sabo L, Lambert LC, McCalman R, Skipper BJ, Davis SM. Assessing food selection in a health promotion program: validation of a brief instrument for American Indian children in the Southwest United States. J Am Diet Assoc. 2000; 100: 205-11. 